\title{
Correlation Between Distal Left Anterior Descending Artery Flow Velocity by Transthoracic Doppler Echocardiography and Corrected TIMI Frame Count Before Mechanical Reperfusion in Patients With Anterior Acute Myocardial Infarction
}

\author{
Souki Lee, MD; Yutaka Otsuji, MD; Shinichi Minagoe, MD; Shuichi Hamasaki, MD; \\ Koichi Toyonaga, MD; Hachiro Obata, MD; Takuro Takumi, MD; \\ Hiroshi Arimura, MD; Masaaki Miyata, MD; Sadatoshi Biro, MD; \\ Hitoshi Toda, MD; Chuwa Tei, MD
}

\begin{abstract}
Background This study was designed to determine the utility of transthoracic Doppler echocardiography (TTDE) in evaluating angiographic Thrombolysis in Myocardial Infarction (TIMI) frame count as a quantitative index of coronary reperfusion in patients with anterior acute myocardial infarction (AMI) before mechanical reperfusion.

Methods and Results Color and pulsed TTDE was performed to evaluate distal left anterior descending coronary artery (LAD) reperfusion in 56 consecutive patients with a first anterior AMI before coronary intervention, and these findings were compared with the corrected TIMI frame count (cTFC) by subsequent angiography. Twenty-four of the 56 patients had LAD reperfusion (TIMI 2 or 3 ) by angiography. Visual antegrade distal LAD flow by color TTDE was detected in 21 of these 24 patients. In the 21 patients, diastolic peak velocity of the distal LAD flow by pulsed TTDE showed a significant correlation with cTFC by angiography $(\mathrm{r}=-0.74$, $\mathrm{p}<0.001$ ). The diagnosis of high risk with angiographic cTFC $>40$ by distal LAD peak velocity $<21 \mathrm{~cm} / \mathrm{s}$ using TTDE had a sensitivity, specificity, and accuracy of $82 \%, 93 \%$, and $91 \%$, respectively.

Conclusion TTDE enables noninvasive and quantitative evaluation of distal LAD reperfusion in patients with anterior AMI in the acute phase before mechanical reperfusion. (Circ J 2005; 69: 1022-1028)
\end{abstract}

Key Words: Acute myocardial infarction; Coronary reperfusion; Echocardiography

Q uick and accurate evaluation of coronary reperfusion in patients with acute myocardial infarction (AMI) is essential to determine whether thrombolysis and/or percutaneous coronary intervention (PCI) is required to achieve reperfusion! ${ }^{1-7}$ Recent advancements in transthoracic Doppler echocardiography (TTDE) have enabled direct visualization and evaluation of coronary blood flow velocity, especially in the distal left anterior descending coronary artery (LAD) ${ }^{8-18}$ This technique has been applied for noninvasive evaluation of Thrombolysis in Myocardial Infarction (TIMI) flow grade, which is widely used as a valuable index of coronary reperfusion. ${ }^{19}$ Our previous study demonstrated that preserved coronary artery flow velocity as determined by TTDE enables differentiation of TIMI 3 from TIMI 0-2 in patients with anterior AMI before PCI ${ }^{20}$ However, the conventional TIMI classification is a semi-quantitative grading of coronary reperfu-

(Received April 20, 2005; revised manuscript received June 9, 2005; accepted June 23, 2005)

Department of Cardiology, Kagoshima City Hospital and Department of Cardiovascular, Respiratory and Metabolic Medicine, Graduate School of Medicine, Kagoshima University, Kagoshima, Japan

Mailing address: Yutaka Otsuji, MD, Department of Cardiovascular, Respiratory and Metabolic Medicine, Graduate School of Medicine, Kagoshima University, 8-35-1 Sakuragaoka, Kagoshima 890-8520,

Japan. E-mail: yutaka@m.kufm.kagoshima-u.ac.jp sion after AMI and is thereby limited by its subjective and categorical nature. TIMI frame count by angiography is a simple, objective, reproducible and quantitative measure of coronary reperfusion ${ }^{21}$ that allows for better prediction of clinical outcomes after AMI22 An intracoronary Doppler guidewire study has demonstrated that distal coronary flow velocity inversely correlates with the TIMI frame count before $\mathrm{PCI}^{23}$ Therefore, we hypothesized that the measurement of LAD flow velocity by TTDE would enable quantitative evaluation of the TIMI frame count by angiography in patients with anterior AMI. To test this hypothesis, we prospectively compared reperfused distal LAD flow velocity by TTDE with the TIMI frame count by angiography in patients with anterior AMI before mechanical reperfusion therapy.

\section{Methods}

Study Group

Between August 2001 and November 2003, 56 consecutive patients at the Department of Cardiology, Kagoshima City Hospital with a first anterior AMI before mechanical reperfusion were enrolled in the study. Inclusion criteria were: (1) typical chest pain lasting $>30 \mathrm{~min}$ and $<12 \mathrm{~h}$ from onset; (2) ST-segment elevation $>0.2 \mathrm{mV}$ in precordial leads; (3) subsequent increase in serum creatine kinase $\geq 2$ - 

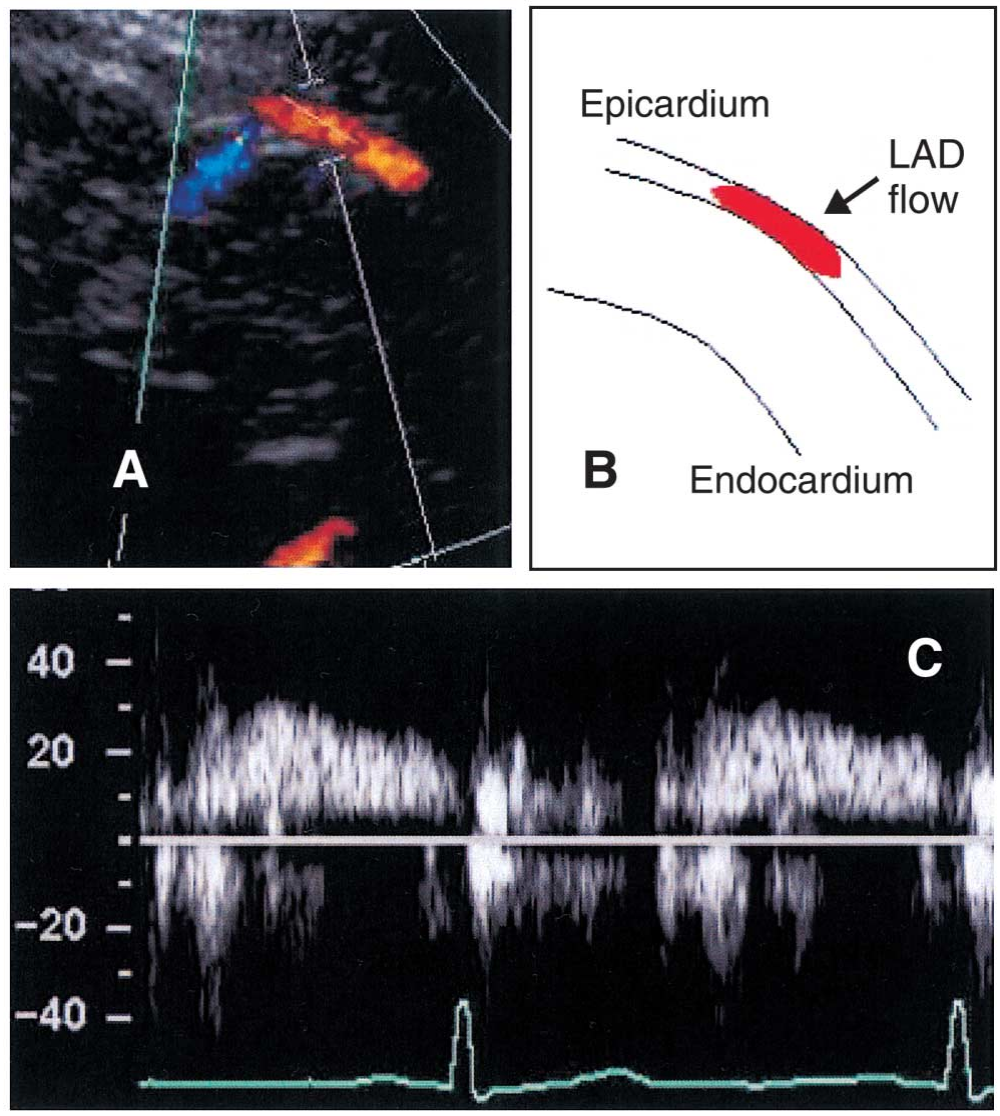

Fig 1. Method of evaluating distal left anterior descending coronary artery (LAD) flow color using pulsed transthoracic Doppler echocardiography (TTDE). (A, B) Distal LAD flow visualized by color TTDE and its tracings. (C) Phasic distal LAD flow velocity by pulsed TTDE. fold the upper limit of normal; and (4) absence of cardiogenic shock, which would otherwise have made assessment of LAD flow problematic. The study protocol was approved by the institutional committee of Kagoshima City Hospital, and each patient gave written, informed consent prior to enrollment.

\section{TTDE}

Before angiography, standard 2-dimensional and Doppler echocardiography (digital ultrasound system HDI-3000, ATL Ultrasound, Bothell, WA, USA) was performed with a $2-3 \mathrm{MHz}$ transducer in the emergency room with patients supine or in the left lateral decubitus position. After the standard echocardiographic examination, distal LAD flow was evaluated with a $4-7 \mathrm{MHz}$ transducer. To image the distal LAD flow, the transducer was placed at the fourth or fifth intercostal space between the cardiac apex and the parasternal area, and the anterior interventricular groove was visualized in the short-axis view. Then, the transducer was rotated to visualize the long axis of the groove, and color flow mapping was applied to visualize the distal LAD flow. Nyquist limit was set in the range of \pm 19 to $\pm 24 \mathrm{~cm} / \mathrm{s}$ to visualize the distal LAD flow with relatively low velocity. When the distal LAD flow was visualized by color flow mapping, its flow velocity was measured by pulsed TTDE, placing a $2.5-\mathrm{mm}$ wide sample volume on the visualized flow signals (Fig 1). Transducer position and direction were adjusted to make the Doppler beam as parallel as possible to the LAD flow, and angle correction was performed for each Doppler measurement. When the distal LAD flow was retrograde by color Doppler or was not visualized within 5 min, antegrade distal LAD flow was considered absent,
Table 1 Baseline Characteristics and Clinical Results $(n=56)$

\begin{tabular}{lc}
\hline \hline Age, years & $66 \pm 13$ \\
M/F & $41 / 15$ \\
Body mass index, $\mathrm{kg} / \mathrm{m}^{2}$ & $22.9 \pm 2.9$ \\
Heart rate, beats/min & $83 \pm 15$ \\
Systolic blood pressure, $\mathrm{mmHg}$ & $130 \pm 20$ \\
Risk factors, $n(\%)$ & \\
Hypertension & $24(43)$ \\
Hypercholesterolemia $(>220 \mathrm{mg} / \mathrm{dl})$ & $20(36)$ \\
Diabetes mellitus & $19(34)$ \\
Current smoking & $28(50)$ \\
Time from onset to TTDE, $h$ & $4.4 \pm 3.0$ \\
Time from TTDE to angiography, min & $29 \pm 11$ \\
IVCT, $n(\%)$ & $18(32)$ \\
LAD culprit lesion & \\
Proximal/distal & $34 / 22$ \\
TIMI flow grade, $n(\%)$ & \\
O & $27(48)$ \\
1 & $4(7)$ \\
2 & $11(20)$ \\
3 & $14(25)$ \\
Collateral & \\
Grade 0-1, $n(\%)$ & $51(91)$ \\
Grade 2, $n(\%)$ & $5(9)$ \\
PCI performed, $n(\%)$ & $48(86)$ \\
Peak creatine kinase, IU/L & $3,979 \pm 2,830$ \\
$Q$-wave infarction, $n(\%)$ & $38(68)$ \\
\hline
\end{tabular}

TTDE, transthoracic Doppler echocardiography; IVCT, intravenous coronary thrombolysis; LAD, left anterior descending coronary artery; TIMI, Thrombolysis in Myocardial Infarction; PCI, percutaneous coronary intervention. 
Table 2 Color Doppler Findings vs Angiographic TIMI Flow Grade

\begin{tabular}{|c|c|c|c|c|c|}
\hline & \multicolumn{3}{|c|}{ Distal LAD reperfusion (-) } & \multicolumn{2}{|c|}{ Distal LAD reperfusion (+) } \\
\hline & $\begin{array}{c}\text { Total } \\
(n=56)\end{array}$ & $\begin{array}{l}\text { TIMI } 0 \\
(n=27)\end{array}$ & $\begin{array}{c}\text { TIMI } 1 \\
(n=4)\end{array}$ & $\begin{array}{l}\text { TIMI } 2 \\
(n=11)\end{array}$ & $\begin{array}{l}\text { TIMI } 3 \\
(n=14)\end{array}$ \\
\hline Visual distal LAD flow (+) & 24 & 3 & 0 & 9 & 12 \\
\hline Visual distal LAD flow (-) & 32 & 24 & 4 & 2 & 2 \\
\hline
\end{tabular}

TIMI, Thrombolysis in Myocardial Infarction; LAD, left anterior descending coronary artery.

and the Doppler coronary flow study was discontinued. All studies were continuously recorded on super-VHS videotape. Observers who were unaware of other data reviewed the Doppler echocardiographic recordings. Pulsed Doppler parameters of LAD flow, including the peak and mean velocity, velocity time integral, and deceleration time of diastolic flow velocity, were measured using the analyzer incorporated in the ultrasound system (Fig 1). Average parameters were calculated from 3 consecutive cardiac cycles.

\section{Coronary Angiography}

After the echocardiographic study, emergency coronary angiography was immediately performed using the femoral arterial technique and $6 \mathrm{~F}$ standard catheters with INNOVA 2000 (GE Medical Systems, Milwaukee, WI, USA). All patients received oral aspirin $(162 \mathrm{mg})$ and intravenous heparin $(5,000 \mathrm{U})$ at the beginning of the procedure. Several views of the LAD were acquired digitally and analyzed quantitatively with a commercially available system (Cardiac QCA, GE Medical Systems). Angiographic lesion parameters, including the percent diameter stenosis, minimum lumen diameter of the culprit lesion, and reference diameter, were automatically computed by a standard method with a contrast-filled $6 \mathrm{~F}$ catheter as a reference. The degree of angiographic collateral circulation was also determined using the Rentrop grading system. ${ }^{24}$

\section{Angiographic TIMI Flow Grade and TIMI Frame Count}

Angiographic TIMI flow grade was evaluated using the initial coronary angiography procedure, as described previously 19 TIMI grade 0 (no perfusion) denoted absent antegrade flow beyond the point of obstruction; TIMI grade 1 (penetration without perfusion) denoted flow beyond the point of obstruction but incomplete filling of the distal vessel; TIMI grade 2 (partial perfusion) represented patent vessels with slow filling and/or slow emptying; TIMI grade 3 (complete perfusion) represented normally brisk flow. TIMI 2 or 3 was defined as the presence of coronary reperfusion. TIMI frame count was also evaluated as an objective and quantitative measure of reperfusion in patients with TIMI grade 2 or 3 . As described previously, ${ }^{21}$ the number of frames from the first frame, in which contrast enters the ostium, to the last frame, when contrast reaches the standardized distal landmark branch, was counted and defined as the TIMI frame count. The distal landmarks included the apical "moustache" branch for the LAD. Because the LAD physiologically requires more frames for the contrast to fill, the TIMI frame counts were corrected by dividing this frame count by 1.7 to derive the corrected TIMI frame count (cTFC). Corrected TFC was measured using a cardiac review station equipped with a frame counter at a rate of 30 frames/s. According to a previous report on risk of inhospital mortality, low-risk and high-risk subgroups were defined as $\mathrm{cTFC} \leq 40$ and $>40$, respectively 22 Observers who were unaware of other patient data reviewed the coronary angiograms.

\section{Reproducibility}

To determine inter-observer variability, 2 observers measured the diastolic peak distal LAD flow velocity by TTDE in 10 patients. Repeated measurements by the same observer were used to assess intra-observer variability. Similarly, inter- and intra-observer variability for the cTFC by angiography was also measured.

\section{Statistical Analysis}

All data are expressed as mean \pm standard deviation. The chi-square test was used to compare the incidence of categorical variables. Continuous variables between 2 groups were compared by the Mann-Whitney U-test. The ability of peak distal LAD flow velocity to indicate cTFC $\leq 40$ was analyzed by receiver operating characteristic (ROC) curves. Statistical analysis was performed with StatView 5.0 software (SAS Institute, Inc, Cary, NC, USA). A p-value $<0.05$ was considered statistically significant.

\section{Results}

\section{Baseline Characteristics and Clinical Results}

Clinical characteristics of the patient population are summarized in Table 1. The mean time from onset to TTDE was $4.4 \mathrm{~h}$, and the mean time from TTDE to angiography was $29 \mathrm{~min}$. Intravenous coronary thrombolysis was performed in 18 patients before the TTDE study, but no thrombolysis was performed between TTDE and angiography. Of the 56 patients, $27(48 \%)$ had TIMI 0, 4 (7\%) had TIMI 1, 11 (20\%) had TIMI 2, and 14 (25\%) had TIMI 3 reperfusion. Forty-eight patients underwent subsequent emergency PCI.

\section{Detection of Coronary Reperfusion by Color TTDE}

By color Doppler echo, antegrade visual distal LAD flow was present in 24 of the 56 patients (Table 2). Of the 24 patients, 12 had TIMI 3, and 9 had TIMI 2. However, the remaining 3 patients had TIMI 0 at the time of angiography. Of the 32 patients without visual distal LAD flow by color TTDE, 28 had TIMI 0 or 1 , and 4 patients had TIMI 2 or 3 . Therefore, the presence of coronary reperfusion with TIMI 2 or 3 grade flow was generally visualized as antegrade distal LAD flow by color Doppler echo. A diagnosis of coronary reperfusion with TIMI 2 or 3 grade flow based on the antegrade visual distal LAD flow by color Doppler echo had a sensitivity, specificity, and accuracy of $86 \%, 88 \%$, and $88 \%$, respectively.

\section{Distal LAD Flow Velocity by Pulsed Doppler vs Angiographic cTFC}

The distal LAD flow velocities by pulsed Doppler echo were evaluated in 21 patients with visual LAD flow by 

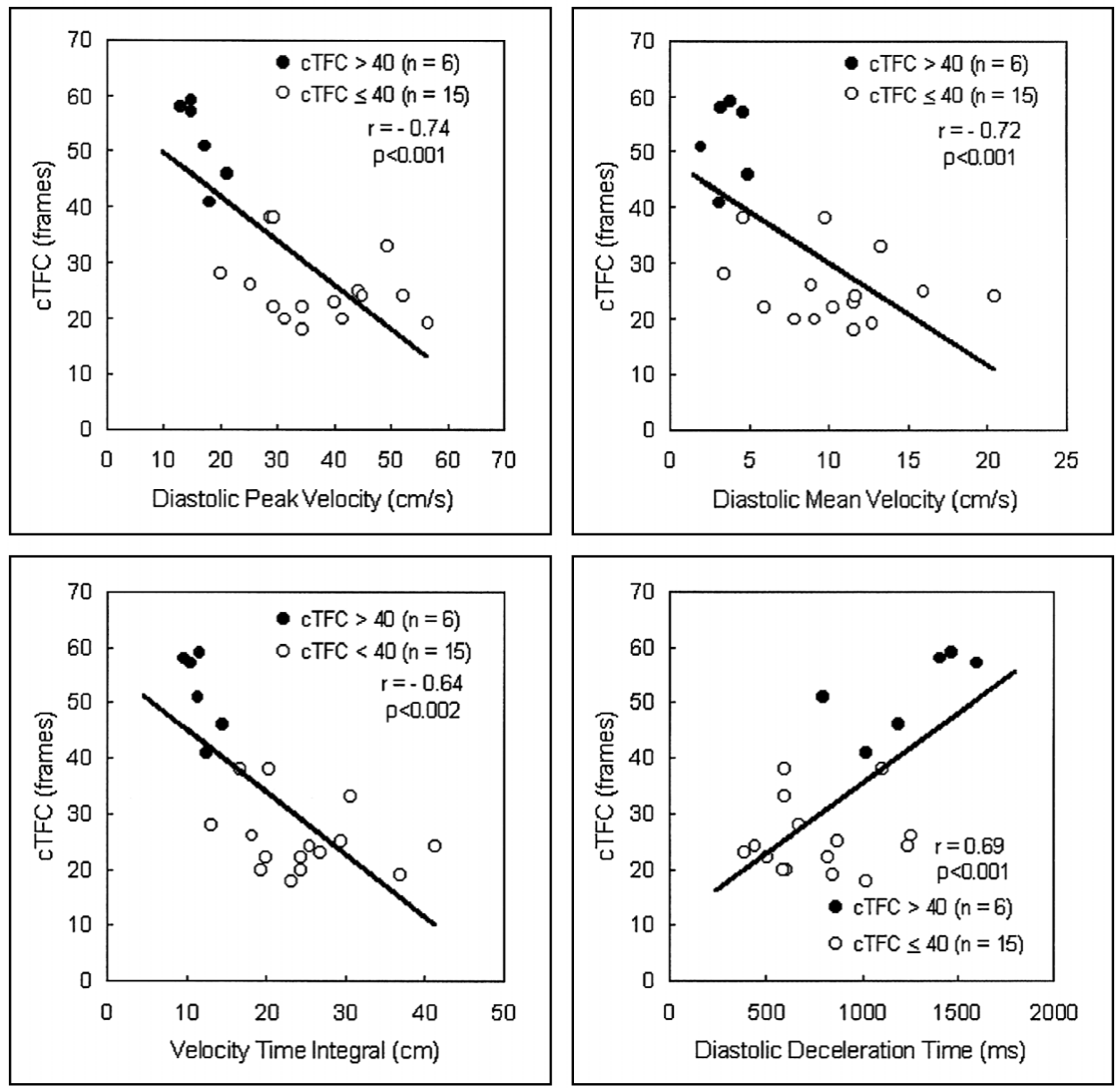

Fig 2. Correlations between diastolic flow velocity profiles by transthoracic Doppler echocardiography (TTDE) and corrected Thrombolysis in Myocardial Infarction frame count (cTFC) by angiography in patients with visual left anterior descending coronary artery reperfusion by color TTDE and TIMI 2 or 3 reperfusion by angiography.

Table 3 Color and Pulsed Doppler Echo Findings vs Angiographic cTFC $\leq$ or $>40$

\begin{tabular}{|c|c|c|c|c|}
\hline & \multirow[b]{2}{*}{$\begin{array}{c}\text { Total } \\
(n=56)\end{array}$} & \multirow{2}{*}{$\begin{array}{c}\text { LAD reperfusion }(-) \\
(n=31)\end{array}$} & \multicolumn{2}{|c|}{$L A D$ reperfusion $(+)$} \\
\hline & & & $\begin{array}{c}c T F C>40 \\
(n=8)\end{array}$ & $\begin{array}{c}c T F C \leq 40 \\
(n=17)\end{array}$ \\
\hline Visual distal LAD flow (+) and $P V \geq 21 \mathrm{~cm} / \mathrm{s}$ & 16 & 1 & 1 & 14 \\
\hline Visual distal LAD flow (-) or $P V<21 \mathrm{~cm} / \mathrm{s}$ & 40 & 30 & 7 & 3 \\
\hline
\end{tabular}

color TTDE and TIMI 2 or 3 by angiography. In these 21 patients, the peak velocity, mean velocity, velocity time integral, and deceleration time of diastolic distal LAD flow by pulsed Doppler echo were all significantly correlated with the cTFC by angiography (Fig 2).

On the basis of ROC curve analysis, a diastolic peak velocity of $21 \mathrm{~cm} / \mathrm{s}$ was chosen as the optimal cutoff value to differentiate cTFC $\leq 40$ (low-risk subgroup) from cTFC $>40$ (high-risk subgroup). The diagnosis of cTFC $\leq 40$ based on the presence of antegrade visual distal LAD flow and a diastolic peak velocity $\geq 21 \mathrm{~cm} / \mathrm{s}$ by color and pulsed TTDE had a sensitivity, specificity, positive predictive value, negative predictive value, and accuracy of $82 \%$, $93 \%, 88 \%, 93 \%$, and $91 \%$, respectively (Table 3 ). Table 4 summarizes the clinical characteristics of patients with cTFC $\leq 40$ and cTFC $>40$. Fig 3 shows representative color and pulsed TTDE with and without angiographic cTFC $\leq 40$.

\section{Reproducibility of Measurements}

The inter-observer variability of the peak diastolic distal LAD flow velocity was $1.1 \pm 0.9 \mathrm{~cm} / \mathrm{s}$ or $4 \pm 4 \%$ of the mean value, and the intra-observer variability was $0.8 \pm 1.0 \mathrm{~cm} / \mathrm{s}$ or $3 \pm 4 \%$ of the mean value. The inter-observer variability of cTFC was $1.7 \pm 1.1$ frames or $5 \pm 4 \%$ of the mean value, and the intra-observer variability was $1.6 \pm 0.7$ frames or $4 \pm 4 \%$ of the mean value.

\section{Discussion}

This study demonstrated that color TTDE-visualized distal LAD flow signal is reasonably sensitive and specific for detection of reperfused LAD flow and that TTDE enables noninvasive diagnosis of the presence or absence of coronary reperfusion in patients with anterior AMI in the acute phase before mechanical reperfusion therapy. In addition, distal LAD reperfused flow velocity by pulsed TTDE significantly correlated with the quantitative severity of LAD reperfusion as determined by angiographic TIMI frame count. This relationship allows for the differentiation of low- and high-risk group with cTFC $\leq$ or $>40$ using the distal LAD flow velocity by noninvasive TTDE. Therefore, TTDE can be used for qualitative as well as quantitative noninvasive evaluation of coronary reperfu- 
Table 4 Profiles of Patients With cTFC $>\mathbf{4 0}$ and cTFC $\leq \mathbf{4 0}$

\begin{tabular}{|c|c|c|c|}
\hline & $\begin{array}{c}c T F C>40 \\
(n=6)\end{array}$ & $\begin{array}{c}c T F C \leq 40 \\
(n=15)\end{array}$ & $p$ value \\
\hline Age, years & $67 \pm 11$ & $64 \pm 15$ & $N S$ \\
\hline$M / F$ & $5 / 1$ & $11 / 4$ & $N S$ \\
\hline Heart rate, beats/min & $87 \pm 17$ & $84 \pm 17$ & $N S$ \\
\hline Systolic blood pressure, $\mathrm{mmHg}$ & $134 \pm 17$ & $122 \pm 15$ & $N S$ \\
\hline Diastolic blood pressure, $\mathrm{mmHg}$ & $78 \pm 12$ & $75 \pm 10$ & NS \\
\hline \multicolumn{4}{|l|}{ Risk factors, $n(\%)$} \\
\hline Hypertension & $2(33)$ & $6(42)$ & $N S$ \\
\hline Hypercholesterolemia $(>220 \mathrm{mg} / \mathrm{dl})$ & $2(33)$ & $5(33)$ & $N S$ \\
\hline Diabetes mellitus & $5(83)$ & $3(20)$ & 0.026 \\
\hline Current smoking & $3(50)$ & $8(53)$ & $N S$ \\
\hline Time from onset to TTDE, $h$ & $5.0 \pm 3.6$ & $4.7 \pm 3.6$ & NS \\
\hline Time from TTDE to angiography, $\mathrm{min}$ & $24 \pm 5$ & $35 \pm 14$ & $N S$ \\
\hline$I V C T, n(\%)$ & $2(33)$ & $8(53)$ & $N S$ \\
\hline \multicolumn{4}{|l|}{ Coronary angiography } \\
\hline Reference diameter, $\mathrm{mm}$ & $2.9 \pm 0.8$ & $2.9 \pm 0.5$ & $N S$ \\
\hline Minimal lumen diameter, mm & $0.3 \pm 0.2$ & $1.1 \pm 0.9$ & 0.012 \\
\hline Diameter stenosis, \% & $91 \pm 7$ & $68 \pm 25$ & 0.043 \\
\hline$c T F C$, frames & $52 \pm 7$ & $26 \pm 6$ & $<0.001$ \\
\hline \multicolumn{4}{|l|}{ Diastolic LAD flow velocity by TTDE } \\
\hline Peak velocity, $\mathrm{cm} / \mathrm{s}$ & $17 \pm 3$ & $36 \pm 10$ & $<0.001$ \\
\hline Mean velocity, $\mathrm{cm} / \mathrm{s}$ & $12 \pm 2$ & $24 \pm 7$ & $<0.001$ \\
\hline Velocity time integral, $\mathrm{cm}$ & $4 \pm 1$ & $10 \pm 4$ & 0.002 \\
\hline Deceleration time, $\mathrm{ms}$ & $1,283 \pm 242$ & $766 \pm 289$ & 0.001 \\
\hline Emergency PCI & $6 / 6$ & $8 / 15$ & $<0.05$ \\
\hline Peak creatine phosphokinase, IU/L & $2,429 \pm 3,742$ & $2,658 \pm 2,116$ & $N S$ \\
\hline$Q$-wave infarction, $n(\%)$ & $3(50)$ & $6(40)$ & $N S$ \\
\hline Cardiac death & $0 / 6$ & $1 / 15$ & $N S$ \\
\hline Congestive heart failure & $0 / 6$ & $2 / 15$ & $N S$ \\
\hline
\end{tabular}

cTFC, corrected Thrombolysis in Myocardial Infarction frame count; NS, no significant difference; TTDE, Transthoracic Doppler echocardiography; IVCT, intravenous coronary thrombolysis; LAD, left anterior descending coronary artery; PCI, percutaneous coronary intervention.

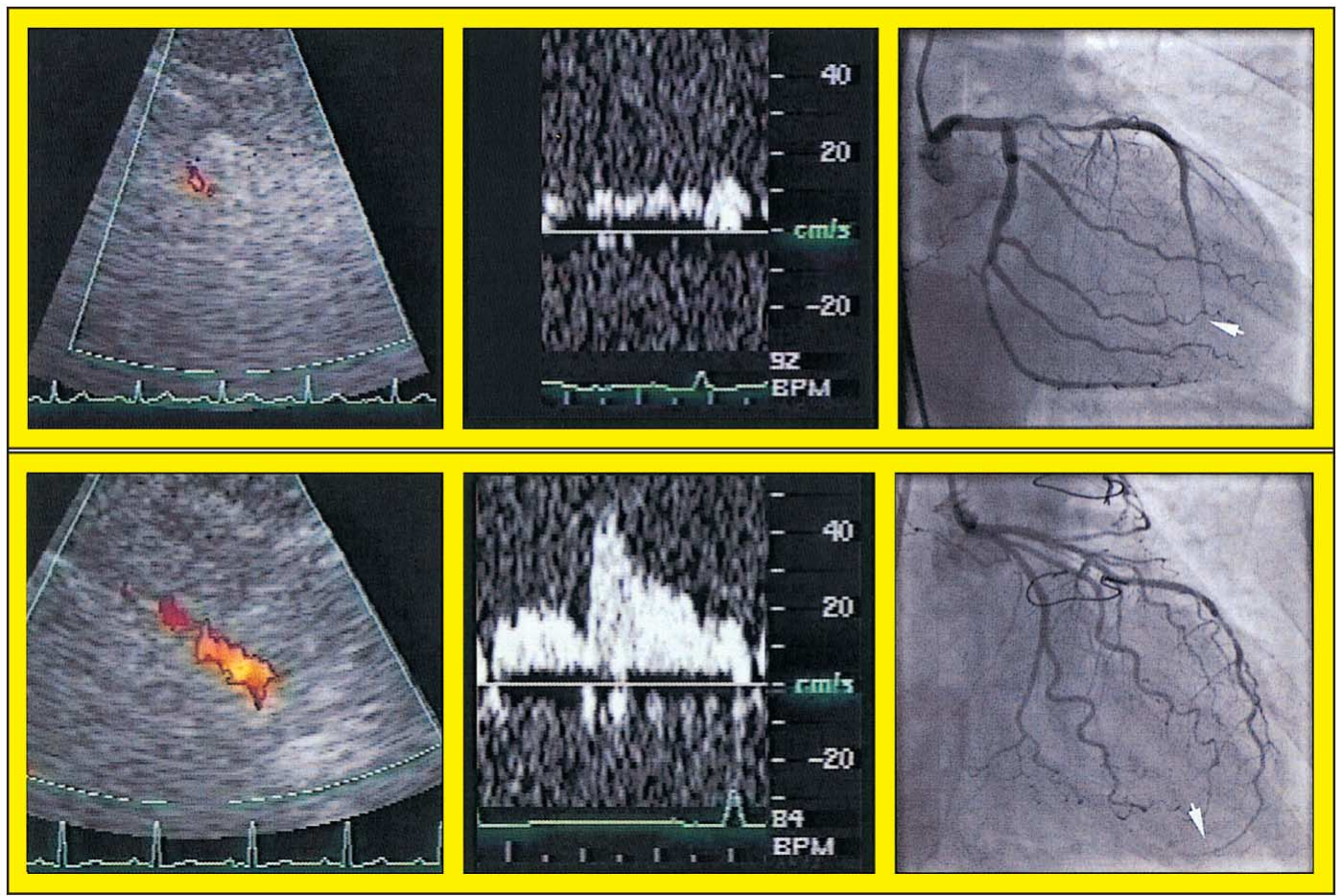

Fig 3. (Upper panel) Patient with color Doppler left anterior descending coronary artery (LAD) reperfused flow signals (Left), but the diastolic peak velocity was low (Middle). Coronary angiography at corrected Thrombolysis in Myocardial Infarction frame count (cTFC) of 40 shows that the contrast has not yet reached the distal landmark branch (Right). The cTFC of this patient was 57 frames. (Lower panel) Patient with color Doppler LAD reperfused flow signals (Left), and the diastolic peak velocity was much higher (Middle). Coronary angiography at cTFC of 24 shows that the contrast has already reached the distal landmark branch (Right). 
sion in patients with anterior AMI before PCI. The results suggest the potential utility of TTDE to determine the indication for emergency angiography and intervention. In combination with other clinical information, a faster distal LAD velocity by TTDE may suggest conservative management without emergency intervention.

\section{Relationship to Previous Studies}

A previous study using an intracoronary Doppler guidewire demonstrated that distal coronary flow velocity inversely correlated with the cTFC before $\mathrm{PCI}^{23}$ which is consistent with the results of the present study. However, the present study extends that finding by demonstrating the utility of noninvasive TTDE in the quantitative evaluation of coronary reperfusion.

Since the time that Fusejima reported the feasibility of TTDE in evaluating coronary flow velocity, TTDE has been applied to many coronary pathophysiologic states, including epicardial coronary stenosis ${ }^{11}, 12$ impaired coronary vasodilatory function, ${ }^{10-13}$ coronary obstruction with collateral flow 17 and intramyocardial small coronary artery flow abnormalities ${ }^{10,18}$ In addition, a recent study has demonstrated that TTDE can be used for semi-quantitative evaluation of coronary reperfusion ${ }^{20}$ The present study confirmed the utility of TTDE in evaluating coronary pathophysiology and further demonstrated its feasibility in the quantitative evaluation of coronary reperfusion and the identification of low- and high-risk patients by objective cTFC.

Slow blood flow or a greater cTFC on angiography may result from reduced coronary flow secondary to severe coronary stenosis, 25,26 which is reflected by diastolic low flow velocity and prolonged diastolic deceleration time in the coronary arteries.27,28 Alternatively, no-reflow secondary to distal embolization of thrombus/plaque components following mechanical reperfusion therapy ${ }^{29-35}$ can also cause slow flow without advanced coronary stenosis. This state is characterized by systolic flow reversal, preserved diastolic peak flow velocity, and rapid diastolic deceleration time? $28,36,37$ Therefore, slow flow or greater cTFC can be associated with either reduced or preserved diastolic flow velocity. In the present study, none of the patients demonstrated a typical no-reflow velocity pattern before mechanical reperfusion, which is consistent with the findings of a previous study? 28 Consequently, the current study demonstrated that (1) diastolic distal LAD flow velocity determined by pulsed TTDE was significantly correlated with cTFC determined by angiography and that (2) the measurement of diastolic peak velocity enables noninvasive differentiation of low-risk (eg, cTFC $\leq 40)$ from high-risk subgroups (eg, cTFC >40). Despite the absence of no-flow in the present patient population, no-reflow can develop in a small number of patients even before mechanical reperfusion, and patients may display a greater cTFC with a preserved diastolic peak velocity. In such cases, the current criteria are problematic, and the diastolic deceleration time may be more useful in detecting high-risk patients with increased cTFC and preserved LAD velocity by noreflow $28,36,37$

\section{Study Limitations}

Patients with right coronary or left circumflex artery disease were excluded from the study because of technical difficulty ${ }^{8-18,20}$ Although administration of contrast agents in patients with AMI is not permitted in Japan, the use of contrast agents to enhance Doppler signals and further technical refinements would expand the utility of TTDE to evaluate the distal $\mathrm{LAD}^{13}$ and other vessels. 38

Some discrepancies in distal LAD reperfusion were noted when comparing TTDE and the subsequent angiography. These discrepancies may be related to the dynamic nature of coronary reperfusion, 39 such as that all 3 patients with visual LAD flow by TTDE, but with TIMI 0 reperfusion by subsequent angiography, showed absent visual flow by TTDE after the angiography. Technical difficulty in detecting adequate distal LAD flow also seems to be a major problem in an emergency situation.

Inclusion in the present study was limited to patients with a first anterior AMI that had developed within $12 \mathrm{~h}$ and with the potential need for emergency $\mathrm{PCI}^{40}$ and the significant relationship between distal peak velocity and cTFC in these patients was caused by the association of greater LAD velocity and favorable reperfusion with a lower cTFC. A higher preserved distal LAD flow velocity might be related to a hyperemic response after reperfusion that can persist for several hours. 41 Therefore, the current method may not be appropriate for use in patients with AMI that is $>12 \mathrm{~h}$ since onset, because reactive hyperemia may have resolved. Diastolic peak distal LAD flow velocity $>21 \mathrm{~cm} / \mathrm{s}$ expressed low risk with cTFC $<40$ in the present study, whereas the LAD velocity $>25 \mathrm{~cm} / \mathrm{s}$ expressed adequate reperfusion with TIMI 3 in a previous study? Further studies are necessary to establish the criteria for the noninvasive evaluation of TIMI 3 or low risk with cTFC $<40$. Although retrospective comparison between the 15 patients with cTFC $<40$ and 6 with cTFC $>40$ showed significant difference in the incidence of emergency PCI, there were no difference in the incidences of cardiac death, congestive heart failure, and progressive left ventricular (LV) remodeling with $20 \%$ or greater increase in LV enddiastolic volume 1 month later compared with that at the acute phase by angiography (2/15 vs $1 / 6$, NS). This suggests that patients with preserved distal LAD velocity or cTFC $<40$ may have less need for emergency PCI. However, a randomized comparison of PCI and conservative treatment without PCI in patients with preserved distal LAD velocity or cTFC $<40$ is required to determine the reliability of the choice to do or not to do PCI based on the preserved distal LAD velocity or cTFC $<40$. Nevertheless, the present study demonstrated that TTDE was of utility in (1) the diagnosis of coronary reperfusion by color flow signals, (2) the quantitative evaluation of coronary reperfusion by distal LAD velocity, and (3) the noninvasive identification of low-risk and high-risk patients as defined by objective cTFC with angiography.

\section{Acknowledgments}

We thank the technical and nursing staff of our cardiac catheterization laboratory and the emergency room staff for their skilled assistance. We also thank Naoko Mizukami, RDCS for her excellent imaging.

\section{References}

1. Anderson JL, Karagounis LA, Becker LC, Sorensen SG, Menlove RL. TIMI perfusion grade 3 but not grade 2 results in improved outcome after thrombolysis for myocardial infarction: Ventriculographic, enzymatic, and electrocardiographic evidence from the TEAM3 study. Circulation 1993; 87: 1829-1839.

2. The GUSTO Angiographic Investigators. The effects of tissue plasminogen activator, streptokinase, or both on coronary-artery patency, ventricular function, and survival after acute myocardial infarction. N Engl J Med 1993; 329: 1615-1622. 
3. Weaver WD, Simes RJ, Betriu A, Grines CL, Zijlstra F, Garcia E, et al. Comparison of primary coronary angiography and intravenous thrombolytic therapy for acute myocardial infarction: A quantitative review. JAMA 1997; 278: 2093-2098.

4. Angioplasty Substudy Investigators. A clinical trial comparing primary coronary angioplasty with tissue plasminogen activator for acute myocardial infarction: The Global Use of Strategies to Open Occluded Coronary Arteries in Acute Coronary Syndromes (GUSTO IIb). N Engl J Med 1997; 336: 1621-1628.

5. Watanabe N, Akasaka T, Fujimoto K, Kajita T, Shigeto F, Neishi Y, et al. Effect of nicorandil, a K+ATP -channel opener, on coronary capillary architecture and volume after early myocardial ischemiareperfusion-a 3-dimensional confocal laser microscopic study. Circ $J$ 2004; 68: 1210-1214.

6. Kurisu S, Inoue I, Kawagoe T, Ishihara M, Shimatani Y, Mitsuba N, et al. Impact of the magnitude of the initial ST-segment elevation on left ventricular function in patients with anterior acute myocardial infarction. Circ J 2004; 68: $903-908$.

7. Watanabe K, Nagao K, Watanabe I, Kanmatsuse K. Relationship between the door-to-TIMI-3 flow time and the infarct size in patients suffering from acute myocardial infarction: Analysis based on the fibrinolysis and subsequent transluminal (FAST-3) trial. Circ J 2004; 68: $280-285$.

8. Fusejima K. Noninvasive measurement of coronary artery blood flow using combined two-dimensional and Doppler echocardiography. $J$ Am Coll Cardiol 1987; 10: 1024-1031.

9. Ross JJ Jr, Mintz GS, Chandrasekaran K. Transthoracic two-dimensional high frequency $(7.5 \mathrm{MHz})$ ultrasonic visualization of the distal left anterior descending coronary artery. J Am Coll Cardiol 1990; 15: $373-377$.

10. Minagoe S, Toyama Y, Niizoe K, Yamaguchi H, Toyoshima S, Umebayashi Y, et al. Transthoracic Doppler echocardiographic detection of intramyocardial coronary artery flow in humans using high frequency transducer. J Cardiol 1997; 30: 149-155.

11. Hozumi T, Yoshida K, Ogata Y, Akasaka T, Asami Y, Takagi T, et al. Noninvasive assessment of significant left anterior descending coronary artery stenosis by coronary flow velocity reserve with transthoracic color Doppler echocardiography. Circulation 1998; 97: $1557-1562$

12. Hozumi T, Yoshida K, Akasaka T, Asami Y, Kanzaki Y, Ueda Y, et al. Value of acceleration flow and the prestenotic to stenotic coronary flow velocity ratio by transthoracic color Doppler echocardiography in noninvasive diagnosis of restenosis after percutaneous transluminal coronary angioplasty. J Am Coll Cardiol 2000; 35: 164-168.

13. Caiati C, Montaldo C, Zedda N, Bina A, Iliceto S. New noninvasive method for coronary flow reserve assessment: Contrast-enhanced transthoracic second harmonic echo Doppler. Circulation 1999; 99: $771-778$.

14. Takeuchi M, Miyazaki C, Yoshitani H, Otani S, Sakamoto K, Yoshikawa J. Assessment of coronary flow-velocity with transthoracic Doppler echocardiography during dobutamine stress echocardiography. J Am Coll Cardiol 2001; 38: 117-123.

15. Shimada K, Watanabe H, Hosoda K, Takeuchi K, Yoshikawa J. Effect of red wine on coronary flow-velocity reserve. Lancet 1999; 354: 1002

16. Otsuka R, Watanabe H, Hirata K, Tokai K, Muro T, Yoshiyama M, et al. Acute effects of passive smoking on the coronary circulation in healthy young adults. JAMA 2001; 286: 436-441.

17. Watanabe N, Akasaka T, Yamaura Y, Akiyama M, Koyama Y, Kamiyama M, et al. Noninvasive detection of total occlusion of the left anterior descending coronary artery with transthoracic Doppler echocardiography. J Am Coll Cardiol 2001; 38: 1328-1332.

18. Voci P, Mariano E, Pizzuto F, Puddu PE, Romeo F. Coronary recanalization in anterior myocardial infarction: The open perforator hypothesis. J Am Coll Cardiol 2002; 40: 1205-1213.

19. The TIMI Study Group. The thrombolysis in myocardial infarction (TIMI) trial: Phase I findings. N Engl J Med 1985; 312: 932-936.

20. Lee S, Otsuji Y, Minagoe S, Hamasaki S, Toyonaga K, Negishi M, et al. Noninvasive evaluation of coronary reperfusion by transthoracic Doppler echocardiography in patients with anterior acute myocardial infarction before coronary intervention. Circulation 2003; 108: $2763-2768$

21. Gibson CM, Cannon CP, Daley WL, Dodge JT, Alexander B, Marble SJ, et al. TIMI frame count: A quantitative method of assessing coronary artery flow. Circulation 1996; 93: 879-888.

22. The Thrombolysis In Myocardial Infarction (TIMI) Study Group. Relationship between TIMI frame count and clinical outcomes after thrombolytic administration. Circulation 1999; 99: 1945-1950.
23. Stankovic G, Manginas A, Voudris V, Pavlides G, Athanassopoulos $\mathrm{G}$, Ostojic M, et al. Prediction of restenosis after coronary angioplasty by use of a new index: TIMI frame count/minimal luminal diameter ratio. Circulation 2000; 101: $962-968$.

24. Rentrop KP, Cohen M, Blanke H, Phillips RA. Changes in collateral channel filling immediately after controlled coronary artery occlusion by an angioplasty balloon in human subjects. J Am Coll Cardiol 1985; 5: 587-592.

25. Zahger D, Karagounis LA, Cercek B, Anderson JL, Sorensen S, Moreno $\mathrm{F}$, et al. Incomplete recanalization as an important determinant of Thrombolysis in Myocardial Infarction (TIMI) grade 2 flow after thrombolytic therapy for acute myocardial infarction. Am J Cardiol 1995; 76: 749-752.

26. The GUSTO-1 Angiographic Investigators. Evolution of early TIMI 2 flow after thrombolysis for acute myocardial infarction. Circulation 1996; 94: 2441-2446.

27. Kern MJ, Moore JA, Aguirre FV, Bach RG, Caracciolo EA, Wolford $\mathrm{T}$, et al. Determination of angiographic (TIMI grade) blood flow by intracoronary Doppler flow velocity during acute myocardial infarction. Circulation 1996; 94: 1545-1552.

28. Akasaka T, Yoshida K, Kawamoto T, Kaji S, Ueda Y, Yamamuro A, et al. Relation of phasic coronary flow velocity characteristics with TIMI perfusion grade and myocardial recovery after primary percutaneous transluminal coronary angioplasty and rescue stenting. Circulation 2000; 101: 2361-2367.

29. Kloner RA, Ganote CE, Jennings RB. The "no-reflow" phenomenon after temporary coronary occlusion in the dog. J Clin Invest 1974; 54: $1496-1508$.

30. Tanaka A, Kawarabayashi T, Nishibori Y, Sano T, Nishida Y, Fukuda D, et al. No-reflow phenomenon and lesion morphology in patients with acute myocardial infarction. Circulation 2002; 105: $2148-2152$.

31. Kotani J, Nanto S, Mintz GS, Kitakaze M, Ohara T, Morozumi T, et al. Plaque gruel of atheromatous coronary lesion may contribute to the no-reflow phenomenon in patients with acute coronary syndrome. Circulation 2002; 106: 1672-1677.

32. Limbruno U, Micheli A, De Carlo M, Amoroso G, Rossini R, Palagi $\mathrm{C}$, et al. Mechanical prevention of distal embolization during primary angioplasty: Safety, feasibility, and impact on myocardial reperfusion. Circulation 2003; 108: 171-176.

33. Kinugasa Y, Ogino K, Furuse Y, Shiomi T, Tsutsui H, Yamamoto T, et al. Allopurinol improves cardiac dysfunction after ischemia-reperfusion via reduction of oxidative stress in isolated perfused rat hearts. Circ J 2003; 67: 781-787.

34. Kyoi S, Otani H, Sumida T, Okada T, Osako M, Imamura H, et al. Loss of intracellular dystrophin: A potential mechanism for myocardial reperfusion injury. Circ J 2003; 67: 725-727.

35. Sugimoto K, Ito H, Iwakura K, Ikushima M, Kato A, Kimura R, et al. Intravenous nicorandil in conjunction with coronary reperfusion therapy is associated with better clinical and functional outcomes in patients with acute myocardial infarction. Circ J 2003; 67: 295-300.

36. Iwakura K, Ito H, Takiuchi S, Taniyama Y, Nakatsuchi Y, Negoro S, et al. Alternation in the coronary blood flow velocity pattern in patients with no reflow and reperfused acute myocardial infarction. Circulation 1996; 94: 1269-1275.

37. Yamamoto K, Ito H, Iwakura K, Kawano S, Ikushima M, Masuyama $\mathrm{T}$, et al. Two different coronary blood flow velocity patterns in Thrombolysis in Myocardial Infarction flow grade 2 in acute myocardial infarction. J Am Coll Cardiol 2002; 40: 1755-1760.

38. Voci P, Pizzuto F, Mariano E, Puddu PE, Chiavari PA, Romeo F. Measurement of coronary flow reserve in the anterior and posterior descending coronary arteries by transthoracic Doppler ultrasound. Am J Cardiol 2002; 90: 988-991.

39. Hackett D, Davies G, Chierchia S, Maseri A. Intermittent coronary occlusion in acute myocardial infarction: Value of combined thrombolytic and vasodilator therapy. N Engl J Med 1987; 317: 10551059.

40. Ryan TJ, Antman EM, Brooks NH, Califf RM, Hillis D, Hiratzka LF, et al. 1999 Update: ACC/AHA guidelines for the management of patients with acute myocardial infarction: Executive summary and recommendations. A report of the American College of Cardiology/American Heart Association task force on practice guidelines (committee on management of acute myocardial infarction). Circulation 1999; 100: 1016-1030.

41. Heyndrickx GR, Amano J, Patrick TA, Manders WT, Rogers GG, Rosendorff $\mathrm{C}$, et al. Effects of coronary artery reperfusion on regional myocardial blood flow and function in conscious baboons. Circulation 1985; 71: 1029-1037. 